\title{
Exophytic Duodenal Stromal Tumor: A Rare Localization
}

\author{
Badr Boutakioute", Meriem Ouali Irissi, Najat Cherif Idrissi El Ganouni
}

Department of Radiology, Mohamed VI Hospital of Marrakech, University of Cadi Ayad Morocco

DOI: $10.36347 /$ sjmcr.2020.v08i02.030

| Received: 10.02.2020 | Accepted: 19.02.2020 | Published: 25.02.2020

*Corresponding author: Badr Boutakioute

\section{Abstract}

Gastrointestinal stromal tumors (GIST) have become a well-defined entity and its classification is no more equivocal, but GIST of duodenum is rarely diagnosed. We report a case of a patient presented with only mild epigastric discomfort. The upper gastrointestinal (GI) endoscopy demonstrated an extrinsic compression of second part of duodenum. Thereafter, CT and MRI of abdomen revealed an exophytic mass emerging from the second part and third of duodenum. The patient underwent a wedge resection of tumor, and the histopathology of the mass confirmed the diagnosis of duodenal stromal tumor. The patient was treated with adjuvant imatinib chemotherapy with no sign of local recurrence after six months of follow up.

Key words: GIST, Duodenum, endoscopy, CT, MRI.

Copyright @ 2020: This is an open-access article distributed under the terms of the Creative Commons Attribution license which permits unrestricted use, distribution, and reproduction in any medium for non-commercial use (NonCommercial, or CC-BY-NC) provided the original author and source are credited.

\section{INTRODUCTION}

Gastrointestinal stromal tumors (GISTs) represent the most common tumor of mesenchymal origin arising in the gastrointestinal tract [1], suggested and recognized as a distinct entity from gastrointestinal smooth-muscle tumors in the 1983 by Mazur and Clark [2]. Despite the fact that these tumors can arise everywhere in the GI tract, the stomach is the most common site of occurrence in more than $50 \%$ of patients while only $3-5 \%$ of GISTs occur in the duodenum [3]. Due to the low incidence and to the complex anatomy of the pancreatico-duodenal region and to the high variability of clinical presentation, the diagnostic assessment and the optimal therapeutic strategy of duodenal GISTs are challenging [4, 5]. This report reviewed the diagnosis, pathology and treatment of a patient of extra mural duodenal GIST.

\section{Case Report}

A 52 years old female presented to us with history of recurrent episode of epigastralgia for last six months. She had no history of vomiting or malena. Clinical examination revealed a mild pale patient with tenderness in epigastric region and no palpable mass. Except for low hemoglobin, her other hemogram and biochemistry were within normal levels. The Upper gastrointestinal (GI) endoscopy demonstrated a chronic gastritis appearance, a prepyloric small ulcer, and an extrinsic compression of second part of duodenum. An abdominal dynamic contrast-enhanced CT scan showed that there was an heterogeneously enhancing relatively defined mass lesion, most likely arising from the second part of duodenum and exophytically extending (Figure 1). There was no definite infiltration into the adjacent organs or metastasis. MRI demonstrated an exophytic mass, involving the second and the third portion of duodenum, measuring $45 \times 60 \times 41 \mathrm{~mm}$, with low signal in T1 weighted images (WI), iso signal in T2 (WI), hight signal on Diffusion (WI) and enhanced heterogeneously after Gadolinium injection, delimiting large areas of necrosis. (Figure 2) These imaging features leded us to evoke the diagnostic of a duodenal GIST.

A laparotomy was performed, and on exploration, there was an exophytic mass arising from second part of duodenum, with no local or distant metastsis or lymphadenopathy. The patient underwent a wedge resection of tumor. The histopathology of the tumor reveals intersecting fasicles of spindle to oval cells. Mitosis are rare about is 5/20 and tumor cells are monomorphic and medium to large size, and there was no lymphatic embolus (Figure 3a-b). The immunohistochemistry analysis highlighted, tumor cells strongly express AML, C- Kit, focally express CD 34 and negative for S100 and Desmin antibodies (Figure 4). The patient had an uneventful hospital course. She was treated with adjuvant imatinib chemotherapy as an outpatient. The Follow-up contrast-enhanced CT scan realized six months after surgical resection shows that there is no evidence of recurrence (Figure 5). 


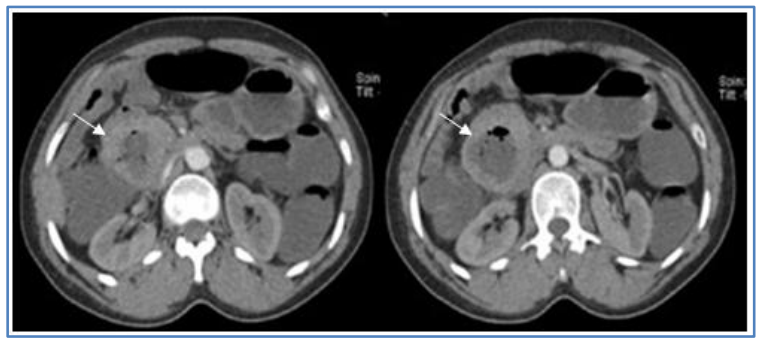

Fig-1: Contrast-enhanced CT scan shows a large, necrotic, exophytic mass (white arrowhead) arising from the second and third portion of duodenum, with a slightly enhancing solid component at the periphery

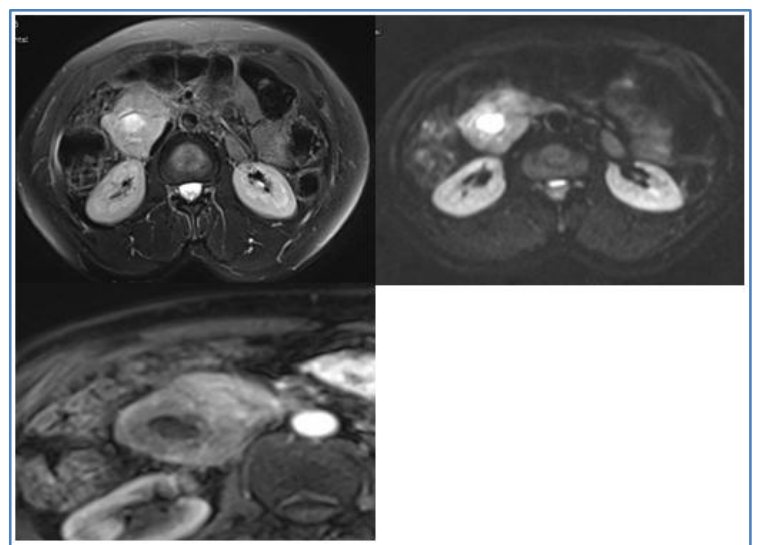

Fig-2: MR images obtained for a preoperative work-up (a) Axial T2-weighted image (b) Axial diffusion-weighted image (DWI), (c) Axial T1-weighted image after Gadolinium injection show an exophytic mass, involving the second and the third portion of duodenum, measuring $45 \times 60 \times 41 \mathrm{~mm}$, hypointense $\mathrm{T} 1$ signal, isointense $\mathrm{T} 2$ (a), hyperintense on diffusion (b) and enhanced heterogeneously after Gadolinium injection (c), delimiting large areas of necrosis.

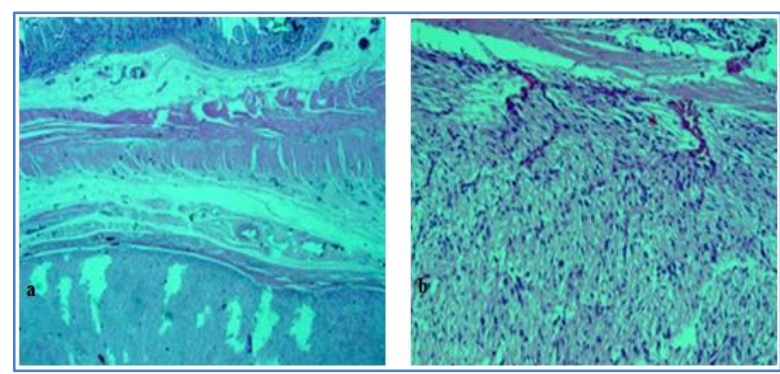

Fig-3: (a) HEx20. Intramuscular round tumor with intestinal mucosa at the surface (b): 40xHE. Well-defined tumor with fusiform cells with few atypias and mitoses

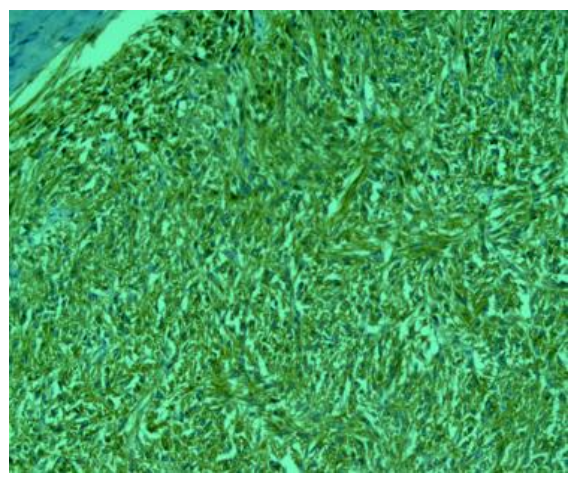

Fig-4: Immunohistochemistry: Tumor cells express CKIT (CD117)

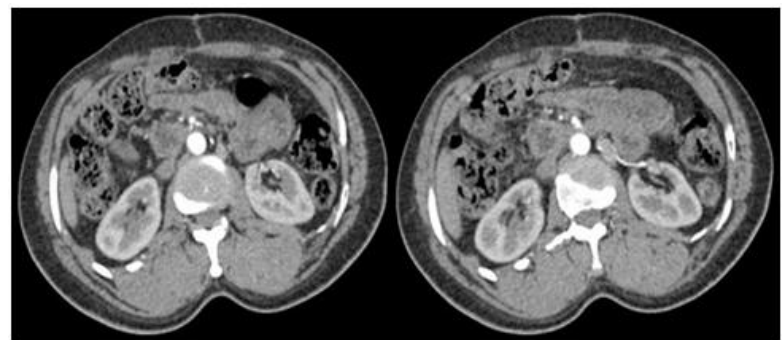

Fig-5: Follow-up contrast-enhanced CT scan obtained 6 months after surgical resection and shows that there is no sign of local recurrence

\section{DISCUSSION}

GISTs are the most common mesenchymal tumors of digestif tract, which mostly occur in the stomach (60\%-70\%) and small intestine (25\%-35\%) [6], followed by the rectum, esophagus, omentum, and mesentery $(<5 \%)$ [4]. Duodenal GISTs are rare lesions, constituting $30 \%$ of primary duodenal tumors and make up only $4.5 \%$ of all GISTs [7]. These tumors are mainly located in the second portion of the duodenum followed by the third, fourth and the first portions [8], and about half of them are malignant [9]. The clinical manifestations of duodenal GISTs are nonspecific and extremely varied. Most GISTs occur sporadically; about $5-10 \%$ occur in hereditary familial syndrome (Type I Neurofibromatosis, Carney triad) [4]. The clinical presentations of duodenal GISTs are highly variable, and they are related to their origin, their size [5], their growth pattern (intramural or extramural), to the presence or absence of mucosal ulceration and location [10]. The gastrointestinal bleeding including melena, hematemesis or symptomatic anemia, and abdominal discomfort or epigastric pain represent the most commonly reported symptoms [11]. Furthermore these tumors rarely cause symptoms and are often diagnosed incidentally, especially for small lesions without mucosal ulceration, or when the tumor growth is extraluminal [12]. Some authors described back pain, jaundice (compression of common bile duct), bowel obstruction, or palpable mass, but these events are very rare [12]. Gastrointestinal endoscopy remains the most common procedure in diagnosing most duodenal GISTs. Even though duodenoscopy are effective to diagnose neoplasms with the typical characteristics of mucosal ulceration or an intramural mass with a centrally ulcerated umbilication $[8,13,14]$, some problems occur for relatively small lesions without the "mass effect" or centrally ulcerated umbilication [13,14]. It allows forceps biopsy whose role, in ambiguous cases, is still controversial because often reveals only normal mucosa adding few data to the GIST management accounting for diagnostic accuracy of approximately $20 \%[15,16]$. Recently, endoscopic ultrasound (EUS) with fine needle aspiration (FNA) to obtain specimens for cytologic examination and immunocytochemical evaluation has been found to be the gold diagnostic standard[17] with high sensitivity and specificity rates $[18,19]$, diagnosing GIST in up to $80 \%$ of patients [19]. EUS can be also performed to 
clarify the original layer of the intramural lesion [20]. Furthermore preoperative histologic diagnosis of GIST might prevent patients from receiving a highly invasive surgical treatment in spite of the theoretical risk for peritoneal seeding of tumor cells by way of the needle tract or tumor rupture during biopsy [21]. Alternative imaging diagnostic modalities include ultrasound, computed tomography (CT), CT Angiography, and magnetic resonance imaging (MRI) that play an important role in duodenal GIST detection and localization. Ultrasonography is often the initial imaging test employed in the investigation of a patient with abdominal pain or mass, it may show a homogenous hypoechoic mass in close relation with the gastrointestinal tract in small GISTs and a vascular mass of mixed echogenicity in large GISTs [22]. But generally it is not an accurate method for the detection of small tumors and has been reported to have a low sensitivity of no more than $26 \%$ [23]. Because of the availability of CT imaging, this method is often used for diagnosing small bowel GIST and can be used for the detection, localization, staging, surgical planning, and in the evaluation of response to therapy, including monitoring postoperative follow-up in patients with GIST [24, 25]. CT generally revealed a small isodense or hypodense round or oval mass with a clear boundary inside the duodenal cavity and slight uniform enhancement. Large tumor appeared as an irregular and heterogeneous enhancement mass with variable areas of cystic degeneration, necrosis, or hemorrhage [16]. Recently, the use of CT Angiography in the diagnosis of GISTs with gastrointestinal bleeding has become feasible, with a reported diagnostic sensitivity of $90.9 \%$ [26, 27]. Magnetic resonance imaging findings are widely variable depending on factors that affect the signal intensity: tumor necrosis, hemorrhage and cavitation [28]. MRI offers good soft tissue contrast and is also an effective imaging modality when differentiating GISTs from other tumors or masses $[29,30]$. Generally the solid components of GISTs show low signal on T1-weighted images and high signal in T2-weighted images with enhancement following gadolinium administration, and signal-intensity voids are present if gas is present within areas of necrotic tumor.

Histologically duodenal GISTs do not differ from other GITSs. These tumors usually present with spindle cell differentiation, which is more frequent than epithelioid cell differentiation or mixed types. There is no apparent correlation between histologic subtype and risk of tumor recurrence $[4,31]$. The mitotic count has been found to be lower in duodenal GISTs, with a median count $<5 / 50$ HPF $[8,32,7]$, which is lower than gastric and small bowel tumors (where a mitotic count $>5 / 50$ HPF has been found in more than $30 \%$ of cases) $[33,9]$.
Duodenal GISTs, as described in the medical literature, do not differ from other GISTs in immunohistochemical reaction, and most of them express CD-117 (c-kit) and CD-34 [8, 32, 7], but it seems that they differ from other GISTs (gastric and small bowel) in the expression of some prognostic factors (ki-67, p53 and p16) [32]. However, these data need to be confirmed by larger series, and there is a probability that a more benign behavior was related to the smaller size and lower mitotic count at diagnosis. For these reasons, duodenal GISTs usually have better prognosis as compared with gastric and small bowel GISTs. According to the National Institutes Health (NIH) risk stratification, they are more frequently identified as very low risk and low risk tumors [32, 34]. The size at diagnosis, prognostic indicators, and risk stratification of duodenal GISTs should guide the surgeon to a limited resection, which should always be a R0 resection. However, up to now, there is still lack of knowledge on the preoperative indicators of bad prognosis and high risk of recurrence. There is no consensus on the optimal surgical treatment for GISTs arising from the duodenum [34]. Operations which vary from tumor enucleation (for extramural GISTs) to pancreaticoduodenectomy for infiltrating or larger tumors have been advocated [8, 5, 32]. Limited resections (LR) can be performed in small tumors not infiltrating the surrounding structures, and when the papilla of Vater can be preserved [8, 35]. In large series, duodenal wedge resection has been performed for small tumors (1-2 $\mathrm{cm}$ in diameter) which were located on the duodenal wall at least $2 \mathrm{~cm}$ from the papilla, so that a safe resection and anastomosis can be carried out with minimal risk of stenosis $[8,35,32]$. Limited resection should only be done when the resection achieves histologically clear margins (R0) and preserves the common bile duct and the papilla of Vater, with an acceptably low risk of perioperative complications. Otherwise pancreaticoduodenectomy should be performed $[8,4,6]$. The disease-free survival rate at 1-3 years after surgical complete resection, in all kinds of resection, has been reported to vary from 86 to $100 \%$ [7, 34], indicating that duodenal GISTs have a slightly more favorable prognosis than gastric or small bowel GISTs.

Imatinib mesylate, a tyrosine kinase inhibitor, plays a key role in the management of GISTs. Its use in neoadjuvant therapy, adjuvant therapy and in tumor recurrence [36, 37], has dramatically changed the natural history of metastatic and recurrent GISTs. In the neoadjuvant setting for GISTs located in the second portion of the duodenum, Imatinib mesylate has been used for tumor downstaging in order to perform a less extensive surgery with free resection margins [7]. However, this requires a precise preoperative diagnosis of GIST which is not always easy to obtain. The use of Imatinib as adjuvant therapy, or for local or metastatic recurrence of duodenal GIST, should not differ from that for other GISTs, and the treatment should be continued indefinitely (usually at a dose of 400 
$\mathrm{mg} /$ day). Interruption of treatment is generally followed by rapid tumor progression $[7,38]$. The major limitation of Imatinib is the development of tumor resistance, which is related to the acquisition of additional c-kit mutations [36]. Though complete surgical resection is feasible in a substantial proportion of GISTs, relapses are common, especially with high-risk GISTs [39]. Surveillance with imaging in these patients can help in timely detection of relapses. The two most common sites of recurrence are the liver and peritoneum. There are no established guidelines for the frequency of surveillance imaging in GIST. In patients who have resectable localized GIST, the The National Comprehensive Cancer Center Network (NCCN) recommends performing CT arbitrarily at intervals of 3-6 months for 3-5 years and then annually in the adjuvant setting after resection of the primary with the aim of detecting local recurrences and distant metastases [40].

\section{CONCLUSION}

Duodenal GISTs are uncommon tumors with uncertain malignant potential. The patient may be asymptomatic or can present with abdominal pain or bleeding. Preoperative diagnosis can be difficult to obtain and the radiologic findings are nonspecific and may represent several entities. Also, the distinction between benign and malignant GISTs cannot be made with radiologic examinations unless metastatic disease or tumor invasion of adjacent structures is depicted. Endoscopic ultrasound and FNA cytology may be helpful in the preoperative setting but the definitive diagnosis of GIST is made immunohistochemically. There is more than one surgical approach available, but the only curative treatment allowing better clinical outcome is complete surgical excision. Preoperative imatinib mesylate can be considered in use in neoadjuvant, adjuvant therapy and in tumor recurrence. Due to the rarity, there are still controversies on the management and behavior of duodenal GISTs

\section{ACKNOWLEDGEMENT}

We thank Dr. Mohamed HAKKOU, for assistance with anatomical images that he provided us, that greatly improved the manuscript.

\section{REFFERENCE}

1. Cavallaro G, Polistena A, D'Ermo G, Pedullà G, De Toma G. Duodenal gastrointestinal stromal tumors: review on clinical and surgical aspects. International Journal of Surgery. 2012 Jan 1;10(9):463-5.

2. Marano L, Boccardi V, Marrelli D, Roviello F. Duodenal gastrointestinal stromal tumor: from clinicopathological features to surgical outcomes. European Journal of Surgical Oncology (EJSO). 2015 Jul 1;41(7):814-22.
3. Kindblom LG, Remotti HE, Aldenborg F, MeisKindblom JM. Gastrointestinal pacemaker cell tumor (GIPACT): gastrointestinal stromal tumors show phenotypic characteristics of the interstitial cells of Cajal. The American journal of pathology. 1998 May;152(5):1259.

4. Beham A, Schaefer IM, Cameron S, von Hammerstein K, Füzesi L, Ramadori G, Ghadimi MB. Duodenal GIST: a single center experience. International journal of colorectal disease. 2013 Apr 1;28(4):581-90.

5. Miki Y, Kurokawa Y, Hirao M, Fujitani K, Iwasa Y, Mano M, Nakamori S, Tsujinaka T. Survival analysis of patients with duodenal gastrointestinal stromal tumors. Journal of clinical gastroenterology. 2010 Feb 1;44(2):97-101.

6. Liu Q, Kong F, Zhou J, Dong M, Dong Q. Management of hemorrhage in gastrointestinal stromal tumors: a review. Cancer management and research. 2018;10:735.

7. Machado NO, Chopra PJ, Al-Haddabi IH, AlQadhi H. Large duodenal gastrointestinal stromal tumor presenting with acute bleeding managed by a whipple resection. A review of surgical options and the prognostic indicators of outcome. JOP. Journal of the Pancreas. 2011 Mar 9;12(2):194-9.

8. Miettinen M, Kopczynski J, Makhlouf HR, Sarlomo-Rikala M, Gyorffy H, Burke A, Sobin LH, Lasota J. Gastrointestinal stromal tumors, intramural leiomyomas, and leiomyosarcomas in the duodenum: a clinicopathologic, immunohistochemical, and molecular genetic study of 167 cases. The American journal of surgical pathology. 2003 May 1;27(5):625-41.

9. Miettinen M, Makhlouf H, Sobin LH, Lasota J. Gastrointestinal stromal tumors of the jejunum and ileum: a clinicopathologic, immunohistochemical, and molecular genetic study of 906 cases before imatinib with long-term follow-up. The American journal of surgical pathology. 2006 Apr $1 ; 30(4): 477-89$

10. Iorio N, Sawaya RA, Friedenberg FK. the biology, diagnosis and management of gastrointestinal stromal tumours. Alimentary pharmacology \& therapeutics. 2014 Jun;39(12):1376-86.

11. Chung JC, Kim HC, Chu CW. Segmental duodenectomy with duodenojejunostomy of gastrointestinal stromal tumor involving the duodenum. Journal of the Korean Surgical Society. 2011 Jun 1;80(Suppl 1):S12-6.

12. Hecker A, Hecker B, Bassaly B, Hirschburger M, Schwandner T, Janßen H, Padberg W. Dramatic regression and bleeding of a duodenal GIST during preoperative imatinib therapy: case report and review. World journal of surgical oncology. 2010 Dec 1;8(1):47.

13. Gervaz P, Huber O, Morel P. Surgical management of gastrointestinal stromal tumours. British Journal of Surgery: Incorporating European Journal of 
Surgery and Swiss Surgery. 2009 Jun;96(6):56778.

14. Mennigen R, Wolters HH, Schulte B, Pelster FW. Segmental resection of the duodenum for gastrointestinal stromal tumor (GIST). World Journal of Surgical Oncology. 2008 Dec $1 ; 6(1): 105$.

15. Sandrasegaran K, Rajesh A, Rushing DA, Rydberg J, Akisik FM, Henley JD. Gastrointestinal stromal tumors: CT and MRI findings. European radiology. 2005 Jul 1;15(7):1407-14.

16. Yang F, Jin C, Du Z, Subedi S, Jiang Y, Li J, Di Y, Zhou Z, Tang F, Fu D. Duodenal gastrointestinal stromal tumor: clinicopathological characteristics, surgical outcomes, long term survival and predictors for adverse outcomes. The American Journal of Surgery. 2013 Sep 1;206(3):360-7.

17. Scarpa M, Bertin M, Ruffolo C, Polese L, D'Amico DF, Angriman I. A systematic review on the clinical diagnosis of gastrointestinal stromal tumors. Journal of surgical oncology. 2008 Oct 1;98(5):384-92.

18. Hoda KM, Rodriguez SA, Faigel DO. EUS-guided sampling of suspected GI stromal tumors. Gastrointestinal endoscopy. 2009 Jun $1 ; 69(7): 1218-23$.

19. Fu K, Eloubeidi MA, Jhala NC, Jhala D, Chhieng DC, Eltoum IE. Diagnosis of gastrointestinal stromal tumor by endoscopic ultrasound-guided fine needle aspiration biopsy-a potential pitfall. Annals of diagnostic pathology. 2002 Oct 1;6(5):294-301.

20. Aydin A, Tekin F, Günşar F, Güler A, Tunçyürek M, Ilter T. Value of endoscopic ultrasonography for upper gastrointestinal stromal tumours. A single center experience. Turk J Gastroenetrol. 2004 Dec;15:233-7.

21. Hong X, Choi H, Loyer EM, Benjamin RS, Trent JC, Charnsangavej C. Gastrointestinal stromal tumor: role of $\mathrm{CT}$ in diagnosis and in response evaluation and surveillance after treatment with imatinib. Radiographics. 2006 Mar;26(2):481-95.

22. O'Sullivan PJ, Harris AC, Ho SG, Munk PL. The imaging features of gastrointestinal stromal tumours. European journal of radiology. 2006 Dec $1 ; 60(3): 431-8$.

23. Masselli G, Casciani E, Polettini E, Laghi F, Gualdi G. Magnetic resonance imaging of small bowel neoplasms. Cancer Imaging. 2013;13(1):92.

24. Werewka-Maczuga A, Osiński T, Chrzan R, Buczek M, Urbanik A. Characteristics of computed tomography imaging of gastrointestinal stromal tumor (GIST) and related diagnostic problems. Polish journal of radiology. 2011 Jul;76(3):38.

25. Lupescu IG, Grasu M, Boros M, Gheorghe C, Ionescu M, Popescu I, Herlea V, Georgescu SA. Gastrointestinal stromal tumors: retrospective analysis of the computer-tomographic aspects. Journal of gastrointestinal and liver diseases. 2007 Jun 1;16(2):147.
26. Martín-Lagos-Maldonado A, Barrientos-Delgado A, Martínez-Tirado M, Casado-Caballero FJ. Angiotomografía helicoidal (angioTAC) en el diagnóstico de una causa poco frecuente de hemorragia digestiva. Revista Española de Enfermedades Digestivas. 2013 Feb;105(2):114-5.

27. Tseng CM, Lin IC, Chang CY, Wang HP, Chen CC, Mo LR, Lin JT, Tai CM. Role of computed tomography angiography on the management of overt obscure gastrointestinal bleeding. PloS one. 2017;12(3).

28. Uchida H, Sasaki A, Iwaki K, Tominaga M, Yada K, Iwashita Y, Shibata K, Matsumoto T, Ohta M, Kitano S. An extramural gastrointestinal stromal tumor of the duodenum mimicking a pancreatic head tumor. Journal of hepato-biliary-pancreatic surgery. 2005 Aug 1;12(4):324-7.

29. Hersh MR, Choi J, Garrett C, Clark R. Imaging gastrointestinal stromal tumors. Cancer control. 2005 Apr;12(2):111-5.

30. Moawad FJ, LaRock TR, Biondi MC, Cash BD, Kurland JE. A case of obscure gastrointestinal bleeding secondary to a small bowel gastrointestinal stromal tumor detected by magnetic resonance enterography. The Medscape Journal of Medicine. 2008;10(11):263.

31. Buchs NC, Bucher P, Gervaz P, Ostermann S, Pugin F, Morel P. Segmental duodenectomy for gastrointestinal stromal tumor of the duodenum. World Journal of Gastroenterology: WJG. 2010 Jun 14;16(22):2788.

32. Yang WL, Yu JR, Wu YJ, Zhu KK, Ding W, Gao Y, Shen QY, Lv KZ, Zhang Q, Yang XJ. Duodenal gastrointestinal stromal tumor: clinical, pathologic, immunohistochemical characteristics, and surgical prognosis. Journal of surgical oncology. 2009 Dec 1;100(7):606-10.

33. Huang HY, Huang WW, Lin CN, Eng HL, Li SH, $\mathrm{Li} \mathrm{CF}$, Lu D, Yu SC, Hsiung CY. Immunohistochemical expression of p16 INK4A, $\mathrm{Ki}-67$, and $\mathrm{Mcm} 2$ proteins in gastrointestinal stromal tumors: prognostic implications and correlations with risk stratification of $\mathrm{NIH}$ consensus criteria. Annals of surgical oncology. 2006 Dec 1;13(12):1633-44.

34. Chung JC, Chu CW, Cho GS, Shin EJ, Lim CW, Kim HC, Song OP. Management and outcome of gastrointestinal stromal tumors of the duodenum. Journal of Gastrointestinal Surgery. 2010 May $1 ; 14(5): 880-3$.

35. Goh BK, Chow PK, Kesavan S, Yap WM, Wong WK. Outcome after surgical treatment of suspected gastrointestinal stromal tumors involving the duodenum: is limited resection appropriate? Journal of surgical oncology. 2008 Apr 1;97(5):388-91.

36. Li G, Tan Y, Yang C, Zhao C, Zhao H, Wang J, Xue Y, Han M, Qian L. Expression and clinical implication of soluble resistance-associated calcium-binding protein gene and multi-drug 
resistance gene in leukemia. Zhonghua zhong liu za zhi [Chinese journal of oncology]. 2002 $\mathrm{Jul}$;24(4):370-4.

37. Demetri GD, Von Mehren M, Blanke CD, Van den Abbeele AD, Eisenberg B, Roberts PJ, Heinrich MC, Tuveson DA, Singer S, Janicek M, Fletcher JA. Efficacy and safety of imatinib mesylate in advanced gastrointestinal stromal tumors. New England Journal of Medicine. 2002 Aug $15 ; 347(7): 472-80$.
38. Pennacchioli E, Colombo C, Berselli M, Gronchi A. Update on management of GIST and postsurgical use of imatinib. Open Access Surgery. 2010;3:63.

39. Call J, Walentas CD, Eickhoff JC, Scherzer N. Survival of gastrointestinal stromal tumor patients in the imatinib era: life raft group observational registry. BMC cancer. 2012 Dec 1;12(1):90.

40. Demetri GD, Baker LH, Benjamin RS. NCCN Soft Tissue Sarcoma Panel Members. NCCN Practice Guidelines in Oncology-V2. 2009. 